\title{
Skyrmion magnetic structure of an ordered FePt monolayer deposited on $\operatorname{Pt}(111)$
}

\author{
S. Polesya, ${ }^{1}$ S. Mankovsky, ${ }^{1}$ S. Bornemann, ${ }^{1}$ D. Ködderitzsch, ${ }^{1}$ J. Minár, ${ }^{1,2}$ and H. Ebert ${ }^{1}$ \\ ${ }^{1}$ Department Chemie/Physikalische Chemie, Ludwig-Maximilians-Universität München, 81377 München, Germany \\ ${ }^{2}$ New Technologies - Research Center, University of West Bohemia, Univerzitni 8, 30614 Pilsen, Czech Republic
}

(Received 23 October 2013; published 22 May 2014)

\begin{abstract}
The effect of the Dzyaloshinsky-Moriya interaction on the magnetic structure of an ordered FePt monolayer deposited on the $\mathrm{Pt}(111)$ surface has been investigated. In the ground state, the pronounced anisotropic geometry of the FePt layer with alternating Fe and Pt chains gives rise to a helimagnetic structure with a strong difference in the helicity period along the chains and perpendicular to them. In the presence of an external magnetic field, the region of stable Skyrmion magnetic structures in the $B-T$ phase diagram has been demonstrated via Monte Carlo simulations using the parameters obtained within first-principles electronic structure calculations. The present study demonstrates clearly that the ratio of the exchange coupling parameters $J / D$ for a deposited magnetic film - being of central importance for the formation of Skyrmions - can be manipulated by growing an overlayer of 2-dimensional compounds with the atoms carrying spontaneous magnetic moments separated by the atoms of nonmagnetic elements.
\end{abstract}

DOI: 10.1103/PhysRevB.89.184414

\section{INTRODUCTION}

The novel topological magnetic structure called Skyrmion crystal (SkX), observed recently in solids, attracts great interest due to various promising physical properties, both from an academic and technological point of view [1-4]. This concerns, in particular, the interaction of Skyrmions (Sk) with an electric current leading to the topological and anomalous Hall effect $[2,3,5,6]$ as well as giving potential access to a new generation of spintronic devices based on current-driven Sk manipulations [4,6]. Predicted and investigated theoretically by Bogdanov et al. [7-13], the SkX requires the presence of chiral interactions in the system, e.g., the spin-orbit coupling (SOC) induced so-called Dzyaloshinsky-Moriya (DM) interaction intrinsic for systems with lack of inversion symmetry [14]. This interaction can be responsible for a helimagnetic (HM) structure in the absence of an external magnetic field $\vec{B}_{\text {ext }}$, while the vortex-like Sk magnetic structure can be stabilized by $\vec{B}_{\text {ext }}[7,8,10,15,16]$. The presence of chiral interactions discerns Skyrmions from so-called magnetic bubbles discussed in the literature and appearing due to dipole-dipole interactions [17]. The SkX state was observed experimentally for the first time in the $\mathrm{MnSi}$ compound with B20 crystal structure [15,18]. Since then, the SkX observation in bulk materials was reported also for other systems, e.g., $\mathrm{FeGe}, \mathrm{Fe}_{1-x} \mathrm{Co}_{x} \mathrm{Si}, \mathrm{Mn}_{1-x} \mathrm{Fe}_{x} \mathrm{Si}$, and $\mathrm{Mn}_{1-x} \mathrm{Co}_{x} \mathrm{Si}$ [19-22]. The conditions for Skyrmion stability in these systems were investigated theoretically by phenomenological considerations based on the Landau-Ginzburg energy functional $[10,15,16,23]$. To describe properly the itinerant-electron properties of magnetism in these systems in the vicinity of the transition to the paramagnetic (PM) state, Bogdanov and Rößler account also for the energy related to longitudinal spin fluctuations. Interesting is that in $3 \mathrm{D}$ materials the temperature window of SkX stability in the phase diagram is rather narrow and located just below the transition temperature to the PM state. In the case of 2D systems, the SkX phase can exist in a larger temperature range approaching the temperature $T=0 \mathrm{~K}[20,23]$.

Note that SkX observation in 3D systems is rather difficult because of the relatively weak DM interaction, while the formation of a noncollinear magnetic state requires the DM value strong enough to compete with the isotropic exchange interactions. This requirement can be met in deposited monolayers as observed experimentally in particular for Fe/Ir(111) overlayers [11], Mn/W(110) overlayers [12] having SOCinduced HM structure, and Sk magnetic structure recently observed in $\mathrm{Fe}(1 \mathrm{ML}) / \mathrm{Ir}(111)$ [24] and $\mathrm{Pd} / \mathrm{Fe}(1 \mathrm{ML}) / \mathrm{Ir}(111)$ [25].

Despite the detailed theoretical investigations based on a phenomenological approach, a complete understanding of the conditions for the SkX appearance cannot be obtained without a detailed analysis of the microscopic origin of the exchange interactions (both isotropic and anisotropic) and magnetocrystalline anisotropy (MCA), that needs a fully relativistic $a b$ initio description of the electronic structure of the systems under consideration. Therefore theoretical schemes based on relativistic density functional theory (DFT) [26] are applied to a large extent in computational simulations to support these experimental efforts [11-13]. In the present work we focus on the first-principles investigation of the formation of the $\mathrm{SkX}$ state in a FePt $(2 \times 1)$ monolayer deposited on $\mathrm{Pt}(111)$, consisting of alternating $\mathrm{Fe}$ and $\mathrm{Pt}$ atomic chains with atoms occupying the Pt crystallographic positions. The geometry of the $(2 \times 1) \mathrm{FePt} / \mathrm{Pt}(111)$ system is depicted in Fig. 1(a).

The magnetic properties of the $(2 \times 1) \mathrm{FePt} / \mathrm{Pt}(111)$ clusters have been recently studied both experimentally and theoretically [27,28], showing the presence of a pronounced noncollinear magnetic structure in the system. This structure is governed by the strong DM interactions as well as a specific atomic structure consisting of alternating $\mathrm{Fe}$ and $\mathrm{Pt}$ chains leading to comparable values of isotropic and DM exchange interactions between neighboring Fe chains. Here we demonstrate that the extension of the cluster to a monolayer leads to the formation of the SkX state in the presence of an external magnetic field.

\section{COMPUTATIONAL DETAILS}

The $T-B$ phase diagram of $\mathrm{FePt} / \mathrm{Pt}(111)$ has been obtained performing Monte Carlo simulations using a standard Metropolis algorithm [29] and based on the extended Heisenberg model accounting for the SOC-induced anisotropy 
(a)

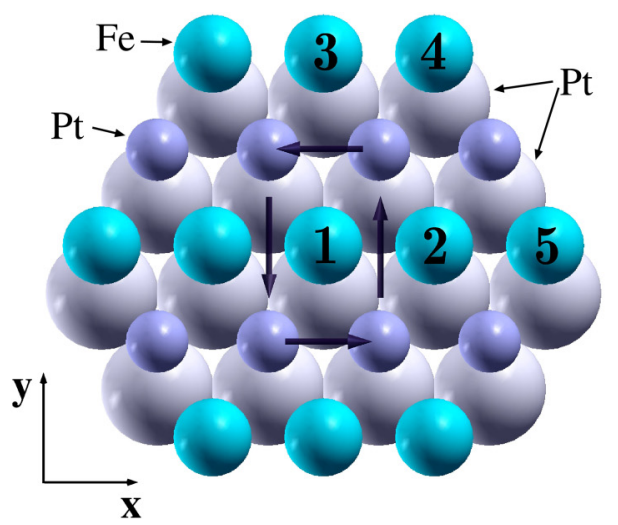

(b)

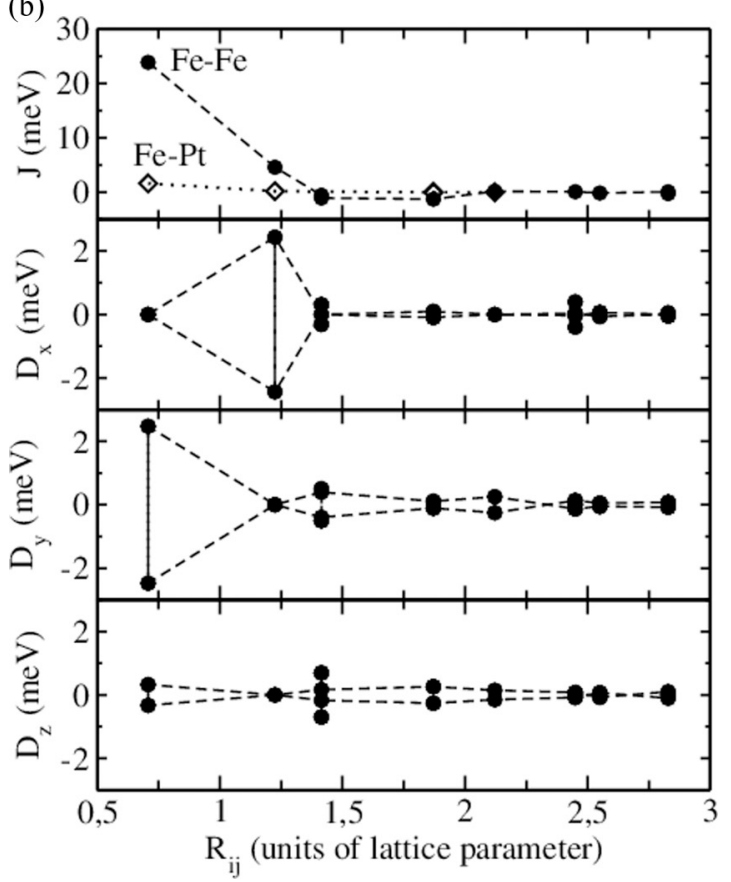

FIG. 1. (Color online) (a) Geometry of the system and directions of in-plane components of DM exchange interactions (shown by the arrows) between $\mathrm{Fe} 1$ and $\mathrm{Fe} 2$ atoms, $\mathrm{Fe} 1$ and $\mathrm{Fe} 3$ atoms, etc. (b) Calculated exchange coupling parameters: isotropic, $J_{i j}$, for $\mathrm{Fe}-\mathrm{Fe}$ (circles) and Fe-Pt (diamonds) (top panel), and $D_{i j}^{x}, D_{i j}^{y}$ and $D_{i j}^{z}$ components of DM interactions between $\mathrm{Fe}$ atoms for $\mathrm{FePt} / \mathrm{Pt}(111)$.

of the exchange interactions. The corresponding Hamiltonian is given by

$$
\begin{aligned}
H= & -\sum_{i, j(i \neq j)} J_{i j} \hat{e}_{i} \cdot \hat{e}_{j}-\sum_{i, j(i \neq j)} \vec{D}_{i j} \cdot\left[\hat{e}_{i} \times \hat{e}_{j}\right] \\
& +\sum_{i} K_{i}\left(\hat{e}_{i}\right)-M \sum_{i}\left(\vec{B}_{\mathrm{ext}} \cdot \hat{e}_{i}\right),
\end{aligned}
$$

with the isotropic exchange coupling parameters $J_{i j}$, the local magnetic moment of atoms $\vec{M}_{i}=M \hat{e}_{i}$, the DM vector $\vec{D}_{i j}$, and the anisotropy constants $K_{i}\left(\hat{e}_{i}\right)$ accounting for the on-site magnetocrystalline anisotropy (MCA) energy associated with each individual moment oriented along $\hat{e}_{i}$.

The main results presented here are obtained taking into account only the exchange interactions between magnetic $\mathrm{Fe}$ atoms, neglecting the contribution from the $\mathrm{Pt}$ atoms having a small induced magnetic moment which gives a small correction to the critical temperature.

A fully relativistic approach for the calculation of the exchange interaction tensor $\underline{J}_{i j}$ based on the magnetic force theorem was used [30]. This gives access to the isotropic and DM exchange coupling parameters used in an extended Heisenberg model, Eq. (1).

The electronic structure calculations for a monolayer of FePt on $\mathrm{Pt}(111)$, denoted FePt/Pt(111), have been performed within the local density approximation for DFT [31], using the spin-polarized relativistic Korringa-Kohn-Rostoker multiple scattering formalism [32]. In this scheme, the Dirac Green's function was calculated self-consistently for $\mathrm{FePt} / \mathrm{Pt}(111)$ assuming pseudomorphic deposition on a Pt slab consisting of 37 atomic layers and having the experimental lattice constant of bulk Pt ( $a=3.924 \AA$ ). All calculations have been performed within the atomic sphere approximation (ASA) to the potentials and lattice relaxations near the surface have been neglected (see Ref. [33] for more details).

\section{DM INTERACTIONS}

The structure of the $(2 \times 1) \mathrm{FePt} / \mathrm{Pt}(111)$ system is depicted in Fig. 1(a) consisting of a monolayer of alternating $\mathrm{Fe}$ and $\mathrm{Pt}$ atomic chains deposited on a $\operatorname{Pt}(111)$ surface. The calculated magnetic moments of the $\mathrm{Fe}$ and $\mathrm{Pt}$ atoms within the $\mathrm{FePt}$ monolayer are $3.14 \mu_{B}$ and $0.22 \mu_{B}$, respectively. The induced magnetic moment in the Pt substrate decreases quickly away from the surface having $0.18 \mu_{B}$ and $0.03 \mu_{B}$ in the interface and next to the interface layers, respectively. Figure 1(b) represents the isotropic exchange coupling parameters $J_{i j}$ and $D_{i j}^{x}, D_{i j}^{y}$, and $D_{i j}^{z}$ components of the DM interaction vector. These parameters for the FePt film on $\mathrm{Pt}(111)$ are very close to those obtained for the $\mathrm{FePt} / \mathrm{Pt}$ (111) cluster [28] (note however the different form of the Heisenberg Hamiltonian used in [28], that leads to the exchange parameters being two times bigger per definition than those used in the present work). Strong $\vec{D}_{i j}$ interactions between the first-neighboring Fe atoms indicate the favorite conditions for the appearance of a HM structure in the system. The properties of $\vec{D}_{i j}$ along different directions $\vec{R}_{i j}$ [Fig. 1(b) and Table I] are clearly governed by the system symmetry $[34,35]$. The symmetry plane between the in-chain Fe atoms 1 and 2 [see Fig. 1(a)] forces the $\vec{D}_{12}$ component along the chain to be equal to 0 . The symmetry plane crossing the $\mathrm{Fe}$ atoms at positions 1 and 3 allows a nonzero component of $\vec{D}_{13}$ interaction along the direction parallel to $\mathrm{Fe}$ and Pt chains. Summarizing the results for the DM interaction for other distances one can conclude that they have pronounced in-plane components (see Fig. 1) responsible

TABLE I. Components of the DM vector $\vec{D}_{i j}$ and the isotropic exchange constant $J_{i j}$ (in $\mathrm{meV}$ ) for the $\mathrm{FePt} / \mathrm{Pt}(111)$.

\begin{tabular}{rcrrrr}
\hline \hline$i-j$ & $R_{i j}$ & $D_{i j}^{x}$ & $D_{i j}^{y}$ & $D_{i j}^{z}$ & \multicolumn{1}{c}{$J_{i j}$} \\
\hline $1-2$ & 0.707 & 0.00 & 2.44 & 0.39 & 23.90 \\
$1-3$ & 1.225 & -2.47 & 0.00 & 0.00 & 4.59 \\
$1-4$ & 1.414 & -0.31 & 0.50 & -0.69 & -0.56 \\
$1-5$ & 1.414 & 0.00 & 0.39 & -0.17 & -1.07 \\
\hline \hline
\end{tabular}


for a rotation of the magnetic moments within a corresponding plane orthogonal to the film. As follows from the model consideration by Fert and Levy [36] giving the expression for DM interactions in the form $\vec{D}_{i j} \sim \frac{\left(\vec{R}_{i n} \cdot \vec{R}_{n j}\right)\left[\vec{R}_{i n} \times \vec{R}_{n j}\right]}{R_{i j} R_{i n} R_{n j}}(j, i$ correspond to the sites of magnetic atoms while $n$ corresponds to the sites of atoms mediating exchange interaction), the $\mathrm{DM}$ interactions are mediated by the Pt atoms and their big magnitude is essentially determined by strong SOC of the $\mathrm{Pt}$ atoms. This allows also drawing conclusions about the small contribution to the $D^{z}$ component of the DM vector at short distances $R_{i j}$, coming (i) from the substrate Pt atoms and (ii) from the $\mathrm{Pt}$ atoms within the $\mathrm{FePt}$ monolayer (this contribution is nonzero only in the presence of the Pt substrate responsible for the breaking of the inversion symmetry). On the other hand, the large magnitude of the $D^{x}$ and $D^{y}$ components is fully determined by the substrate $\mathrm{Pt}$ atoms, giving evidence to the crucial role of their strong SOC values for the in-plane components of the $\vec{D}_{i j}$ interactions between the Fe atoms. At large distances $R_{i j}$ all three components have the same order of magnitude, due to contributions of many Pt atoms involved into the mediation of Fe-Fe DM exchange interactions. Thus, one can clearly see a pronounced SOC-induced effect in the present system leading to DM couplings strong enough to compete with the isotropic exchange coupling and to create a pronounced noncollinear magnetic structure.

\section{HELIMAGNETIC STRUCTURE}

Magnetic torque calculations show a rather strong in-plane MCA of $1.1 \mathrm{meV}$ per Fe atom with the magnetic easy axis being perpendicular to the $\mathrm{Fe}$ and $\mathrm{Pt}$ chains. The MCA energy was taken into account in all present MC simulations based on Heisenberg Hamiltonian Eq. (1). First, the calculations have been performed in the absence of an external magnetic field. The resulting noncollinear magnetic structure obtained at $T=1 \mathrm{~K}$ is presented in Fig. 2. The period of the helicity in the continual model represented by Landau-Ginzburg energy functional is determined by the ratio of exchange stiffness and Dzyaloshinsky-Moriya constants, $A / D$ [8]. In general, these values are represented by tensors of first rank, $D^{\alpha}=$ $\left|\sum_{j} \vec{D}_{0 j} R_{0 j}^{\alpha}\right|$, and second rank, $A^{\alpha \beta}=\sum_{j} J_{0 j} R_{0 j}^{\alpha} R_{0 j}^{\beta}$ [37], characterizing a spatial anisotropy of the system, that is important for the present case having $C_{s}$ symmetry. In particular, these values give the anisotropy of the energy of spin-wave excitations with wave vector $\vec{q}=q \hat{n}_{\alpha}$ along different directions, $\hat{n}_{\alpha}=\hat{x}, \hat{y}: \omega\left(q^{\alpha}\right)=\tilde{A}^{\alpha \alpha}\left(q_{\alpha}\right)^{2}+\tilde{D}^{\alpha} q_{\alpha}+\omega_{0}$, with spin-wave coefficients $\tilde{A}^{\alpha \alpha}=\frac{2 \mu_{B}}{M} A^{\alpha \alpha}$ and $\tilde{D}^{\alpha}=\frac{2 \mu_{B}}{M} D^{\alpha}$, and $\omega_{0}$ the spin-wave gap created by magnetocrystalline anisotropy. The strong anisotropy of the exchange stiffness tensor is well recognized considering the first-neighbor isotropic exchange

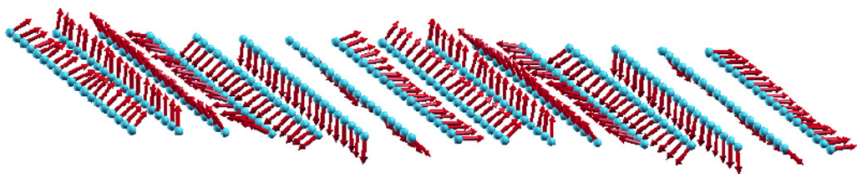

FIG. 2. (Color online) Helimagnetic structure in FePt/Pt(111). The arrows represent the magnetic moments of $\mathrm{Fe}$ atoms. coupling parameters (see Table I) within and between the Fe chains. This leads to a complex HM structure with a different period along different directions; i.e., the period along the chains is essentially longer than perpendicular to the Fe chains, as can be clearly seen in Fig. 2. Raising the temperature from $T=0 \mathrm{~K}$, the magnetization of the system exhibits two phase transition: from HM to FM state at $T_{H}=40 \mathrm{~K}$, and from FM to the paramagnetic (PM) at $T_{C}=90 \mathrm{~K}$.

\section{SKYRMION MAGNETIC STRUCTURE}

The presence of an external magnetic field $\vec{B}_{\text {ext }}$ perpendicular to the surface plane, exceeding a certain critical value, gives rise to the formation of Skyrmions in the system under consideration. The distribution of the magnetic moments within the single Skyrmion obtained using the MC simulation is shown in Fig. 3. The topological quantum number (or winding number) calculated for such a magnetic texture is equal to -1 (see Appendix), being a property of a single Skyrmion (see, e.g., [18,38]). One can clearly see the rather small Skyrmion size (compared with the period of the HM structure) due to a small $A / D$ ratio. Its different size in two orthogonal directions within the plane is determined by the spatial anisotropy of the exchange coupling parameters, $J_{i j}$ and $\vec{D}_{i j}$. The in-plane tangential components of the magnetic moments governed by the $D_{i j}^{z}$ component of DM interactions are close to 0 , in line with the discussions by Rößler et al. [16] on the magnetic moment distribution within the Skyrmions in the FM system with $C_{n v}$ symmetry.

Analyzing the requirements for the Skyrmion formation for $\mathrm{FePt} / \mathrm{Pt}(111)$, one can use rather simple qualitative arguments. In this consideration the effect of a demagnetizing field can be neglected being small in the case of a magnetic monoatomic overlayer. A competition of isotropic exchange and DM interactions determine the period of the HM structure without magnetic field and anisotropy by minimizing the energy of the system. In the presence of an out-of-plane magnetic field the minimization of the Zeeman energy is realized by minimizing the $2 \mathrm{D}$ area with magnetic moments opposite to the direction of the magnetic field [the Zeeman energy gain is shown by yellow color, while the energy loss is shown by blue in Fig. 3(a)]. However, this costs the energy originating from the interatomic isotropic exchange, due to a noncollinear orientation of the in-plane components of the magnetic moments within the Skyrmion [see Fig. 3(b), where the exchange energy loss is shown by blue color], assuming that the Skyrmion size is close to the period of the HM structure. The competition of these two factors determines the conditions for the formation of Skyrmions at some critical value of the external magnetic field $\vec{B}_{\text {ext }}$. Despite the strong simplification neglecting the role of other contributions (due to exchange interactions and MCA) to the SkX energetics, this qualitative consideration visualizes the role of the magnetic field for the $\mathrm{SkX}$ formation as a ground state $(T=0 \mathrm{~K})$, relevant in the case $2 \mathrm{D}$ system.

As was mentioned above, the 2D anisotropy of the system leads to a corresponding shape of the Skyrmions obtained via calculations accounting for $\mathrm{Fe}-\mathrm{Fe}$ exchange interactions only, as soon as only Fe atoms carry spontaneous magnetic moments weakly dependent on magnetic configuration. Since isotropic exchange interactions between $\mathrm{Fe}$ atoms within 

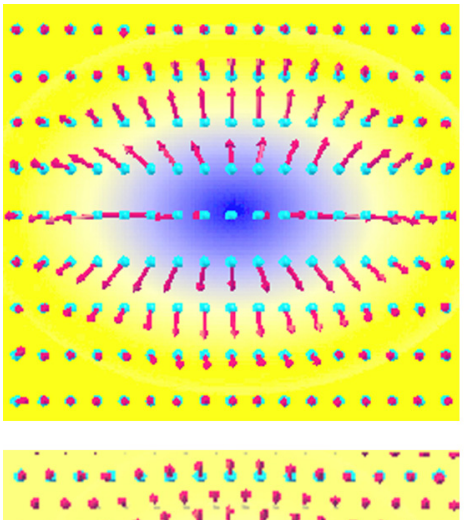

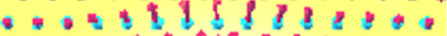

$\because t^{2} t^{2} t^{2}$

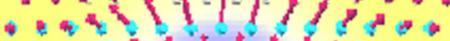

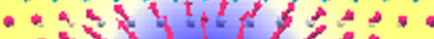

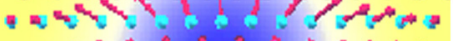

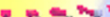

$4=0$

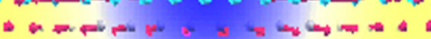

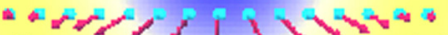

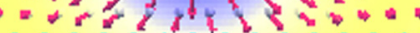

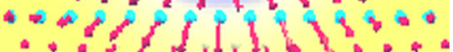

(15) 50

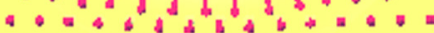

.

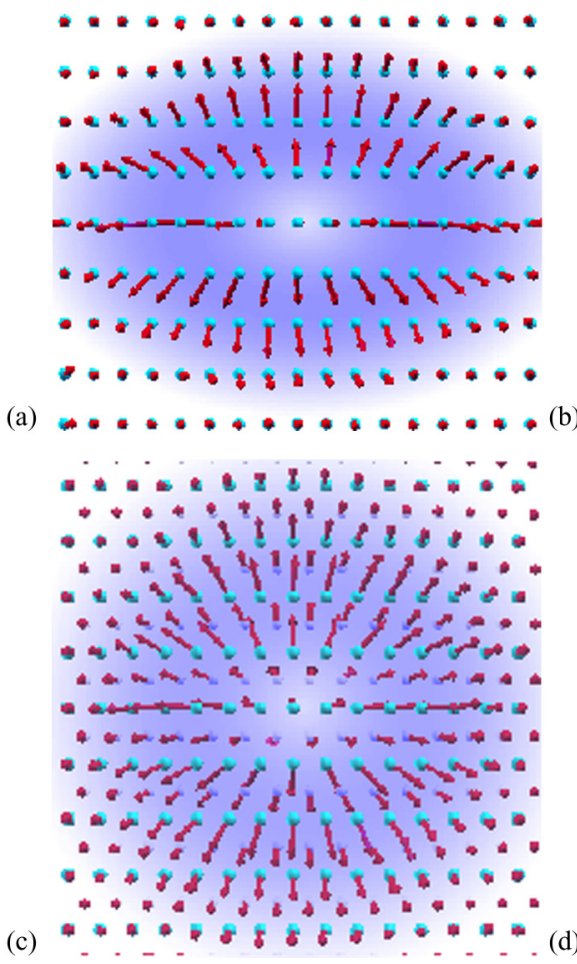

FIG. 3. (Color online) Magnetic moment distribution within the Skyrmion. Yellow and blue colors in (a) represent schematically the region giving gain and loss of Zeeman energy in the presence of a magnetic field; blue color in (b) shows the region giving loss of the exchange energy contributed by in-plane components of magnetic moments. (c) and (d): Structure of single Sk obtained with the contributions of the Fe-Pt exchange interactions taken into account [the same color code as in (a) and (b)]. Long arrows show the spontaneous magnetic moments on $\mathrm{Fe}$ atoms; short arrows indicate the induced magnetic moments on Pt atoms. the $\mathrm{Fe}$ chains are much stronger than the interchain $\mathrm{Fe}-\mathrm{Fe}$ interactions, their competition with DM interactions results in the weaker modulation of magnetic structure along the chains when compared to the direction perpendicular to the chains. However, in systems containing atoms with induced magnetic moments, i.e., $\mathrm{Pt}$ in the present case, the $\mathrm{Fe}-\mathrm{Pt}$ interactions, $J_{i j}^{\mathrm{Fe}-\mathrm{Pt}}$, can lead to an additional contribution to the Fe-Fe exchange energy if the $J_{i j}^{\mathrm{Fe}-\mathrm{Pt}}$ (shown in Fig. 1) value is not negligible (see discussions, e.g., [39-41]). However, to account for this contribution in MC calculations is not straightforward and is rather time consuming. Therefore, we have performed here the calculations accounting for $\mathrm{Fe}-\mathrm{Pt}$ interactions only to investigate their effect on the shape of the Skyrmion, following the scheme described in Ref. [41]. Based on this, we conclude that the Pt contribution could modify the phase diagram, but these changes should not be crucial. The result, obtained at $T=1 \mathrm{~K}$, is presented in Fig. 3(c). One can see that the Skyrmion shape in this case is more symmetric. This occurs due to an additional Fe-Pt exchange contribution competing with DM interactions and resulting in the increase of the effective $J / D$ (see the above discussion) ratio along the direction perpendicular to the Fe chains, and leading that way to the increase of the Skyrmion size along the direction perpendicular to the Fe chains.

\section{PHASE DIAGRAM}

The magnetic structure of $\mathrm{FePt} / \mathrm{Pt}(111)$ and its behavior at different temperature and external magnetic field, perpendicular to the surface, has also been investigated via Monte Carlo simulations, using the standard Metropolis algorithm. For this, only the interactions within the Fe subsystem with well-defined local magnetic moments have been taken into account. The MC unit cell containing $2500 \mathrm{Fe}$ atoms was extended using periodic boundary conditions. The in-plane magnetic anisotropy was taken into account with MCA direction perpendicular to Fe chains and $E_{\mathrm{MCA}}=1.1 \mathrm{meV}$ (obtained in the present calculations). The important role of the magnetic anisotropy for the stabilization of the SkX state in some systems was discussed in the literature $[10,42]$. Therefore, a set of additional MC calculations has been performed for the out-of-plane MCA direction (with the MCA energy equal to $1.1 \mathrm{meV}$ ) as well as for the MCA energy set to 0 . All these results exhibit rather small differences indicating a weak effect of the MCA and therefore the main responsibility of the exchange interactions (isotropic and DM) for the SkX stabilization in the system under consideration. Therefore, we present below only the results obtained for in-plane MCA found in the present DFT calculations.

More results of the MC simulation can be seen in Fig. 4 representing the low-temperature part of the $T-B$ phase diagram. The critical temperatures within the MC simulations have been obtained from an analysis of the heat capacity $c_{v}(T, B)$ and susceptibility $\chi(T, B)$. Figure 5 shows the temperature dependence of these parameters for three different values of the magnetic field: $B=12 \mathrm{~T}$ (top panel), $B=8 \mathrm{~T}$ (middle panel), and $B=4 \mathrm{~T}$ (bottom panel). In the first two cases the $c_{v}(T)$ curves have only one maximum corresponding to the boundary of the SkX region, while the two maxima of $c_{v}(T)$ in the case of $B=4 \mathrm{~T}$ are associated with the lowand high-temperature boundaries of the SkX region shown in Fig. 4 in red. To improve the statistical error a series of independent calculations have been performed here for each point at the phase diagram (i) by varying the temperature $T$ at a fixed magnetic field $B$ value and (ii) by varying the magnetic field, keeping the temperature constant. Nevertheless, one has to admit that perfect phase boundaries have not been obtained. As soon as the density of Skyrmions reproduces the SkX phase boundaries with the same accuracy as $c_{v}(T, B)$ and $\chi(T, B)$, the phase diagram is plotted representing the density of Skyrmions 

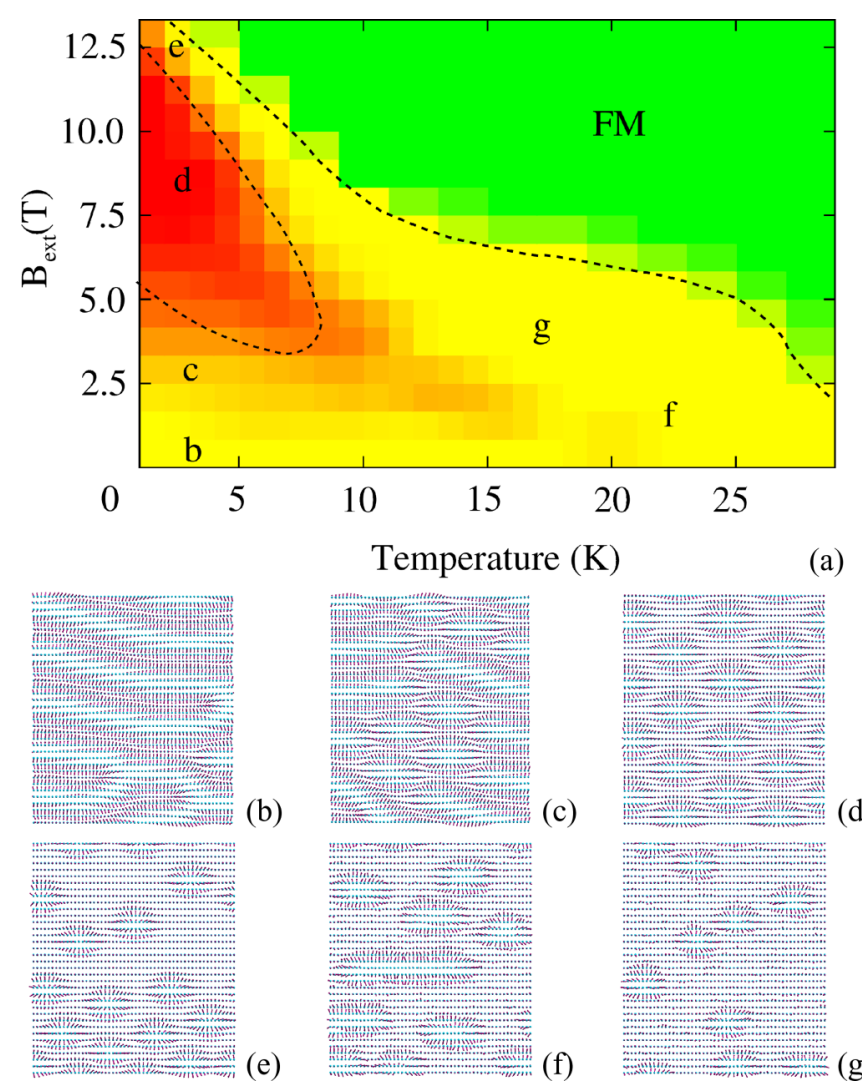

FIG. 4. (Color online) (a) Low-temperature part of $B-T$ phase diagram calculated for $\mathrm{FePt} / \mathrm{Pt}(111)$; (b)- $(\mathrm{g})$ representative magnetic structures of the phase diagram regions indicated in (a) obtained at $B=0.0 \mathrm{~T}, T=3.0 \mathrm{~K}(\mathrm{~b}), B=2.5 \mathrm{~T}, T=3.0 \mathrm{~K}(\mathrm{c}), B=7.5 \mathrm{~T}$, $T=3.0 \mathrm{~K}(\mathrm{~d}), B=12.5 \mathrm{~T}, T=3.0 \mathrm{~K}(\mathrm{e}), B=1.0 \mathrm{~T}, T=22.0 \mathrm{~K}$ (f), $B=3.5 \mathrm{~T}, T=17.0 \mathrm{~K}(\mathrm{~g})$.

at each $(B, T)$ point. Without magnetic field the system exhibits a rather pronounced SOC-induced HM structure [Fig. 4(b)] below the critical temperature $T_{H}=40 \mathrm{~K}$. The FM structure is stabilized in the temperature region from $T_{H}=40 \mathrm{~K}$ to $T_{C}=90 \mathrm{~K}$, while the following temperature increase drives the system into the PM state. The critical temperatures have been evaluated from the temperature-dependent behavior of the magnetic susceptibility, using the average magnetization in the system as an order parameter. An external magnetic field, $\vec{B}_{\text {ext }}$, leads at low temperature to the formation of Skyrmions coexisting with the HM structure [Fig. 4(c)]. Raising of the magnetic field at low temperature leads to the formation of a Skyrmionic lattice [Fig. 4(d)]. The density of Skyrmions is shown in the phase diagram by red and yellow, representing the SkX area with highest Skyrmion density by red, while the wide region with low Skyrmion density is colored in yellow. Note that the boundary between the low Skyrmion (yellow) and FM (green) phases was plotted using only the data on the temperature dependence of Skyrmion density which is very low in this region. The dashed lines are drawn as a guide for the eyes to visualize the approximate boundaries separating different phases. At high magnetic fields and low temperature the concentration of Skyrmions is reduced resulting in the mixed FM + Sk state [Fig. 4(e)] until the transition to the FM state. At these values of the magnetic field the transition to the

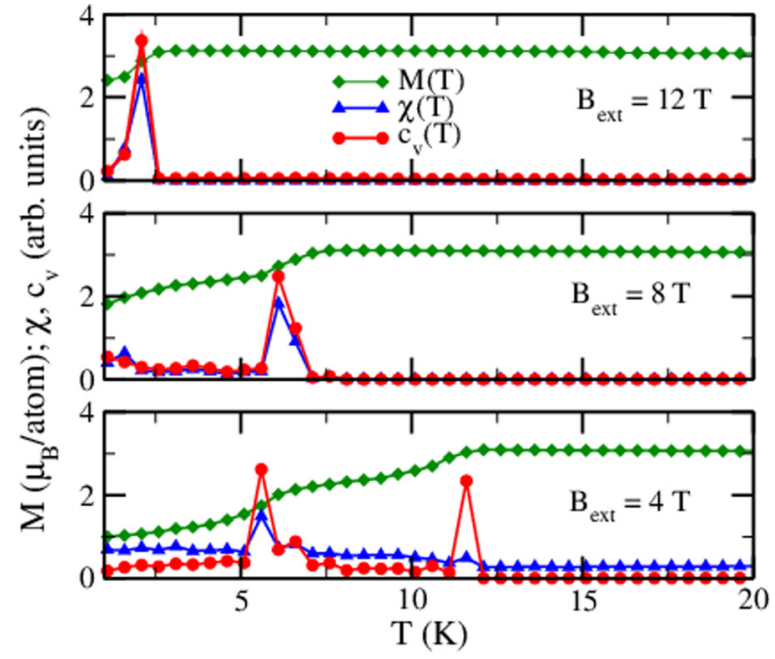

FIG. 5. (Color online) Temperature-dependent magnetization $M(T)$ (diamond), susceptibility $\chi(T)$ (triangles), and heat capacity $c_{v}(T)$ functions represented for three different magnetic fields: $B=12 \mathrm{~T}$ (top panel), $B=8 \mathrm{~T}$ (middle panel), and $B=4 \mathrm{~T}$ (bottom panel).

$\mathrm{FM}+\mathrm{HM}+\mathrm{Sk}$ state does not occur. On the other hand, at high temperature and low magnetic field, as it was discussed above, a mixed FM + HM + Sk state is observed [Fig. 4(f)], while an increasing of the magnetic field leads to a mixed FM + Sk state [Fig. 4(g)]. At all values of the magnetic field the temperature increase leads to a transition from the noncollinear magnetic state to the FM state.

Thus, the behavior of the magnetic structure of FePt/Pt(111) in the presence of a finite external field shows striking differences when compared with the properties of 3D systems, e.g., FeGe, MnSi, MnGe, etc. This is, first of all, due to the well-defined Fe local magnetic moment indicating a small effect of longitudinal fluctuations on the formation of Skyrmionic magnetic properties. A Skyrmion lattice state in the phase diagram appears at low temperature, in contrast to 3D systems, where the Skyrmion lattice formation occurs close to the temperature of the transition to the disordered magnetic state. Because the Curie temperature in the system is rather high, the temperature increase leads first to the transition from the Skyrmionic to the FM state and then from the FM to the PM state, in contrast to the SkX-PM transition observed in the 3D bulk compounds FeGe, MnSi, and MnGe. Finally, we would like to stress that strong in-plane components of the $\vec{D}_{i j}$ interactions, governed by the substrate $\mathrm{Pt}$ atoms in $\mathrm{FePt} / \mathrm{Pt}(111)$, lead to a small Sk size (see above), that makes this system attractive for technological applications. Note however that too strong DM interactions can lead to a stabilization of the SkX as a ground state [24,43], even without external magnetic field. One can expect different behavior of this state when compared to those discussed above, although detailed investigations of its phase diagram so far have not been done.

\section{ACKNOWLEDGMENTS}

Financial support by the DFG via SFB 689 (Spinphänomene in reduzierten Dimensionen) is gratefully acknowledged. 


\section{APPENDIX}

To calculate the winding number for an isolated Skyrmion obtained within MC calculations, its magnetic configuration $\hat{n}\left(\vec{R}_{i}\right)=\vec{m}\left(\vec{R}_{i}\right) /\left|\vec{m}\left(\vec{R}_{i}\right)\right|$ can be represented by a continuous model in cylindrical coordinates. In this case, the direction of the magnetic moment is equal to $\hat{n}(\vec{r})=\left(\left|\vec{n}_{||}\right| \cos \phi,\left|\vec{n}_{||}\right| \sin \phi,\left|\vec{n}_{z}\right|\right)=(\sin \theta \cos \phi, \sin \theta \sin \phi, \cos \theta)$ for each point $\vec{r}=(\rho \cos \phi, \rho \sin \phi)$ in the $x y$ (film) plane. Note that the in-plane component of the magnetic moment is always aligned along the radius $\hat{\rho}: \vec{n}_{\|}=\left(\left|\vec{n}_{\|}(\rho)\right| \cos \phi\right.$, $\left.\left|\vec{n}_{\|}(\rho)\right| \sin \phi\right)||(\rho \cos \phi, \rho \sin \phi)$, and $\theta(\rho)$ is an angle with respect to the $z$ axis, depending only on the distance from the center of the Skyrmion. In this case the winding number can be calculated by doing the necessary integration using polar coordinates:

$$
\begin{aligned}
W & =\frac{1}{4 \pi} \int d x \int d y \hat{n} \cdot\left[\frac{\partial \hat{n}}{\partial x} \times \frac{\partial \hat{n}}{\partial y}\right] \\
& =\frac{1}{4 \pi} \int_{0}^{R_{s}} \rho d \rho \int_{0}^{2 \pi} d \phi\left[\frac{\partial \hat{n}}{\partial x} \times \frac{\partial \hat{n}}{\partial y}\right] .
\end{aligned}
$$

Taking into account the expressions for the derivatives in polar coordinates (see, e.g., [44]) and assuming that $\theta(r=$ $0)=\pi$ and $\theta\left(r=R_{s}\right)=0$ (where $R_{s}$ is the Skyrmion radius) one obtains the winding number according to the shape of the Skyrmion:

$$
W=\frac{1}{4 \pi} \int_{0}^{R_{s}} \rho d \rho\left[\frac{1}{\rho} \frac{\partial \theta}{\partial \rho} \sin \theta\right] \int_{0}^{2 \pi} d \phi=-1 .
$$

[1] A. N. Bogdanov and U. K. Rößler, Phys. Rev. Lett. 87, 037203 (2001).

[2] M. Lee, W. Kang, Y. Onose, Y. Tokura, and N. P. Ong, Phys. Rev. Lett. 102, 186601 (2009).

[3] T. Schulz, R. Ritz, A. Bauer, M. Halder, M. Wagner, C. Franz, C. Pfleiderer, K. Everschor, M. Garst, and A. Rosch, Nat. Phys. 8, 301 (2012).

[4] F. Jonietz, S. Mühlbauer, C. Pfleiderer, A. Neubauer, W. Münzer, A. Bauer, T. Adams, R. Georgii, P. Böni, R. A. Duine, K. Everschor, M. Garst, and A. Rosch, Science 330, 1648 (2010).

[5] A. Neubauer, C. Pfleiderer, B. Binz, A. Rosch, R. Ritz, P. G. Niklowitz, and P. Böni, Phys. Rev. Lett. 102, 186602 (2009).

[6] Y. Li, N. Kanazawa, X. Z. Yu, A. Tsukazaki, M. Kawasaki, M. Ichikawa, X. F. Jin, F. Kagawa, and Y. Tokura, Phys. Rev. Lett. 110, 117202 (2013).

[7] A. N. Bogdanov and D. A. Yablonsky, Sov. Phys. JETP 68, 101 (1989).

[8] A. Bogdanov and A. Hubert, J. Magn. Magn. Materials 138, 255 (1994).

[9] U. K. Rößler, A. N. Bogdanov, and C. Pfleiderer, Nature (London) 442, 797 (2006).

[10] A. B. Butenko, A. A. Leonov, U. K. Rößler, and A. N. Bogdanov, Phys. Rev. B 82, 052403 (2010).

[11] K. von Bergmann, S. Heinze, M. Bode, E. Y. Vedmedenko, G. Bihlmayer, S. Blügel, and R. Wiesendanger, Phys. Rev. Lett. 96, 167203 (2006).

[12] M. Bode, M. Heide, K. von Bergmann, P. Ferriani, S. Heinze, G. Bihlmayer, A. Kubetzka, O. Pietzsch, S. Blügel, and R. Wiesendanger, Nature (London) 447, 190 (2007).

[13] M. Heide, G. Bihlmayer, and S. Blügel, Phys. Rev. B 78, 140403 (2008).

[14] I. E. Dzyaloshinskii, Sov. Phys. JETP 19, 960 (1964).

[15] C. Pappas, E. Lelièvre-Berna, P. Falus, P. M. Bentley, E. Moskvin, S. Grigoriev, P. Fouquet, and B. Farago, Phys. Rev. Lett. 102, 197202 (2009).

[16] U. K. Rößler, A. A. Leonov, and A. N. Bogdanov, J. Phys.: Conf. Series 200, 022029 (2010).

[17] N. S. Kiselev, A. N. Bogdanov, R. Schäfer, and U. K. Rößler, J. Phys.: Appl. Phys. 44, 392001 (2011).

[18] S. Mühlbauer, B. Binz, F. Jonietz, C. Pfleiderer, A. Rosch, A. Neubauer, R. Georgii, and P. Böni, Science 323, 915 (2009).
[19] X. Z. Yu, Y. Onose, N. Kanazawa, J. H. Park, J. H. Han, Y. Matsui, N. Nagaosa, and Y. Tokura, Nature (London) 465 , 901 (2010).

[20] X. Z. Yu, N. Kanazawa, Y. Onose, K. Kimoto, W. Z. Zhang, S. Ishiwata, Y. Matsui, and Y. Tokura, Nat. Mater. 10, 106 (2010).

[21] A. Bauer, A. Neubauer, C. Franz, W. Münzer, M. Garst, and C. Pfleiderer, Phys. Rev. B 82, 064404 (2010).

[22] W. Münzer, A. Neubauer, T. Adams, S. Mühlbauer, C. Franz, F. Jonietz, R. Georgii, P. Böni, B. Pedersen, M. Schmidt, A. Rosch, and C. Pfleiderer, Phys. Rev. B 81, 041203 (2010).

[23] J. H. Han, J. Zang, Z. Yang, J.-H. Park, and N. Nagaosa, Phys. Rev. B 82, 094429 (2010).

[24] S. Heinze, K. von Bergmann, M. Menzel, J. Brede, A. Kubetzka, R. Wiesendanger, G. Bihlmayer, and S. Blügel, Nat. Phys. 7, 713 (2011).

[25] N. Romming, C. Hanneken, M. Menzel, J. E. Bickel, B. Wolter, K. von Bergmann, A. Kubetzka, and R. Wiesendanger, Science 341, 636 (2013).

[26] A. H. MacDonald and S. H. Vosko, J. Phys. C: Solid State Phys. 12, 2977 (1979).

[27] J. Honolka, T. Y. Lee, K. Kuhnke, A. Enders, R. Skomski, S. Bornemann, S. Mankovsky, J. Minár, J. Staunton, H. Ebert, M. Hessler, K. Fauth, G. Schütz, A. Buchsbaum, M. Schmid, P. Varga, and K. Kern, Phys. Rev. Lett. 102, 067207 (2009).

[28] S. Mankovsky, S. Bornemann, J. Minár, S. Polesya, H. Ebert, J. B. Staunton, and A. I. Lichtenstein, Phys. Rev. B 80, 014422 (2009).

[29] K. Binder, Rep. Prog. Phys. 60, 487 (1997).

[30] L. Udvardi, L. Szunyogh, K. Palotás, and P. Weinberger, Phys. Rev. B 68, 104436 (2003).

[31] S. H. Vosko, L. Wilk, and M. Nusair, Can. J. Phys. 58, 1200 (1980).

[32] H. Ebert, in Electronic Structure and Physical Properties of Solids, Vol. 535 of Lecture Notes in Physics, edited by H. Dreyssé (Springer, Berlin, 2000), p. 191

[33] S. Bornemann, O. Šipr, S. Mankovsky, S. Polesya, J. B. Staunton, W. Wurth, H. Ebert, and J. Minár, Phys. Rev. B 86, 104436 (2012).

[34] T. Moriya, Phys. Rev. 120, 91 (1960). 
[35] A. Crépieux and C. Lacroix, J. Magn. Magn. Mater. 182, 341 (1998).

[36] A. Fert and P. M. Levy, Phys. Rev. Lett. 44, 1538 (1980).

[37] A. I. Liechtenstein, M. I. Katsnelson, and V. A. Gubanov, J. Phys. F: Met. Phys. 14, L125 (1984).

[38] Y. Onose, Y. Okamura, S. Seki, S. Ishiwata, and Y. Tokura, Phys. Rev. Lett. 109, 037603 (2012).

[39] O. N. Mryasov, Phase Transitions 78, 197 (2005).

[40] M. Ležaić, P. Mavropoulos, J. Enkovaara, G. Bihlmayer, and S. Blügel, Phys. Rev. Lett. 97, 026404 (2006).
[41] S. Polesya, S. Mankovsky, O. Šipr, W. Meindl, C. Strunk, and H. Ebert, Phys. Rev. B 82, 214409 (2010).

[42] U. K. Rößler, A. A. Leonov, and A. N. Bogdanov, J. Phys.: Conf. Series 303, 012105 (2011).

[43] Y.-Q. Li, Y.-H. Liu, and Y. Zhou, Phys. Rev. B 84, 205123 (2011).

[44] D. Varshalovich, A. Moskalev, and V. Khersonskii, Quantum Theory of Angular Momentum: Irreducible Tensors, Spherical Harmonics, Vector Coupling Coefficients, 3 Nj Symbols (World Scientific Publishing, Singapore, 1988). 